\title{
Evaluación da la actividad de tres enzimas proteolíticas como biocatalizadores lácteos
}

\section{Activity Evaluation of three proteolytic enzymes as dairy biocatalysts}

Josselyn Gabriela Bermeo Berrones. ${ }^{1}$, Iván Patricio Salgado Tello. ${ }^{2}$, Cesar Iván Flores Mancheno. ${ }^{3}$ \& Tatiana Elizabeth Sánchez Herrera. ${ }^{4}$

\begin{abstract}
.
DOI: $\underline{\text { https://doi.org/10.33262/concienciadigital.v3i2.1.1231 }}$

The study of proteolytic enzymes at an industrial level is of great importance since their catalytic utility may be used especially in the dairy industry, so the objective of the study was to obtain three proteolytic enzymes from pineapple (Annanas-cosmosus L), papaya (Carica-papaya) and fig (Ficus-carica L), for which different latex extraction techniques were used, applying a completely random design and Tukey as a statistical test, with 5 repetitions and one size of an experimental unit of $5 \mathrm{~kg}$, resulting in highly significant differences between the three enzymes studied, while the methods of mechanical breakdown for pineapple, longitudinal cuts for papaya and fig were used to obtain the latex. Physicochemical evaluations of 7.62 Brix and $5.48 \mathrm{pH}$ are obtained for papaya, 12.12 Brix and $2.20 \mathrm{pH}$ for pineapple and 7.24 Brix with $6.48 \mathrm{pH}$ for fig, while that, in what corresponds to the yield, it can be mentioned that with $18 \mathrm{~g} / \mathrm{Kg}$ ficin is the enzyme with the best result. For the enzymatic activity, a completely randomized design with a unit size of $0.0375 \mathrm{mg}$ of enzyme was used, using milk as a substrate for the catalysis process, reporting values of $.15 .74 \mathrm{U} / \mathrm{mg}$ protein with a time of $73.92 \mathrm{sec}$ for bromelain, $15.09 \mathrm{U} / \mathrm{mg}$ protein with a clotting time of $61.44 \mathrm{sec}$ for papain and $14.87 \mathrm{U} / \mathrm{mg}$ protein with a time of 58 seconds for
\end{abstract}

${ }^{1}$ Profesional independiente, Riobamba, Ecuador. josslyn@yahoo.es

${ }^{2}$ Escuela Superior Politécnica de Chimborazo, Facultad de Ciencias Pecuarias. Riobamba, Ecuador. ivan.salgado@espoch.edu.ec

${ }^{3}$ Escuela Superior Politécnica de Chimborazo, Facultad de Ciencias Pecuarias. Riobamba, Ecuador. ifloresm1@yahoo.es

${ }^{4}$ Escuela Superior Politécnica de Chimborazo, Facultad de Ciencias Pecuarias. Riobamba, Ecuador. tatiana.sanchez@espoch.edu.ec 
ficin, indicating that the results show highly significant differences and demonstrating that Ficin is the enzyme that has an effective and efficient enzyme speed and clotting time.

Keywords: Proteolytic enzymes, papaya, fig, pineapple, dairy, enzymatic activity

\section{Resumen.}

El estudio de las enzimas proteolíticas a nivel industrial tiene una gran importancia ya que su utilidad catalítica podrá ser utilizada especialmente en la industria láctea, por lo que el objetivo del estudio fue la obtención de tres enzimas proteolíticas a partir de la piña (Annanas-cosmosus L), papaya (Carica-papaya) y el higo (Ficus-carica L), para lo cual se utilizaron técnicas diferentes de extracción del látex, aplicando un diseño completamente al azar y Tukey como prueba estadística, contando con 5 repeticiones y un tamaño de unidad experimental de $5 \mathrm{~kg}$, dando como resultado diferencias altamente significativas entre las tres enzimas estudiadas, en tanto que, para la obtención del látex se utilizaron los métodos de la ruptura mecánica para la piña, cortes longitudinales para la papaya e higo. Se obtiene valoraciones físico-químicas de 7,62 Brix y 5,48 de $\mathrm{pH}$ para la papaya, 12,12 Brix y 2,20 de $\mathrm{pH}$ de para la piña y 7,24 Brix con $6,48 \mathrm{pH}$ para el higo, mientras que, en lo que corresponde al rendimiento se puede mencionar que con $18 \mathrm{~g} / \mathrm{Kg}$ la ficina es la enzima con mejor resultado. Para la actividad enzimática se empleó un diseño completamente al azar con un tamaño de unidad de $0,0375 \mathrm{mg}$ de enzima utilizando leche como sustrato para el proceso de catálisis reportándose valores de, 15,74 U/mg proteína con un tiempo de 73,92seg para la bromelina, $15,09 \mathrm{U} / \mathrm{mg}$ proteína con un tiempo de coagulación de $61,44 \mathrm{seg}$ para la papaína y $14,87 \mathrm{U} / \mathrm{mg}$ proteína con un tiempo de 58 segundos para la ficina indicando que los resultados presentan diferencias altamente significativas y demostrando que la ficina es la enzima que presenta una velocidad enzimática y tiempo de coagulación eficaces y eficientes.

Palabras claves: Enzimas proteolíticas, papaya, higo, piña, lácteo, actividad enzimática

\section{Introducción.}

Durante los últimos años menciona (Bermeo, 2019, citado en Dalgo, 2012), la biotecnología ha venido experimentando grandes avances en aplicaciones industriales, debiéndose a la demanda de productos de calidad, así como también procesos industriales óptimos, esto ha incentivado el estudio de nuevas tecnologías para lograr dichas metas obteniendo así beneficios tanto para la industria como para el consumidor.

Las enzimas son moléculas de proteínas que tienen la capacidad de facilitar y acelerar las reacciones químicas, disminuyendo el nivel de la "energía de activación" propia de la reacción. Se entiende por energía de activación al valor de la energía que es necesario aplicar (en forma de calor, electricidad o radiación) para que las moléculas determinadas colisionen y se produzca una reacción química entre ellas como lo indica, (Bermeo, 2019, citado en Del Moral et al., n.d.).

Las enzimas indica (Bermeo, 2019, citado en Mauricio et al., n.d.) son altamente específicas en cada reacción catalizada y en sus cambios de reactantes, los cuales son llamados sustratos. Una enzima generalmente cataliza una reacción simple o un grupo de reacciones estrechamente ligadas. Reacciones laterales que conducen al derroche de subproductos rara vez ocurren en las reacciones 
catalizadas al contrario de la sin catalizar.

La clasificación de las enzimas se encuentra normalizada por las reacciones que brindan en los diferentes procesos de catálisis, teniendo seis principales categorías de clasificación:

Las Oxidoreductasas comenta (Bermeo, 2019, citado en Alba et al., 2008) catalizan reacciones de óxido reducción. Precisan la colaboración de las coenzimas de óxido reducción, que aceptan o ceden los electrones correspondientes; tras la acción catalítica, estas coenzimas quedan modificadas en su grado de oxidación, por lo que deben ser transformadas antes de volver a efectuar la reacción catalítica.

Las transferasas enzimas de este tipo permiten el cambio de grupos específicos de una molécula a otra. Transfieren grupos activos (obtenidos de la ruptura de ciertas moléculas) a otras sustancias receptoras. Suelen actuar en procesos de Inter conversión de monosacáridos, aminoácidos (Bermeo, 2019, citado en Alba et al., 2008).

Las hidrolasas permiten romper moléculas de alto peso molecular, como reacciones de hidrólisis con la consiguiente obtención de monómeros a partir de polímeros. Actúan en la digestión de los alimentos, previamente a otras fases de su degradación (Bermeo, 2019, citado en Alba et al., 2008).

(Bermeo, 2019, citado en Alba et al., 2008) menciona que las isomerasas actúan sobre determinadas moléculas obteniendo de ellas sus isómeros de función o de posición. Suelen actuar en procesos de Inter conversión.

En lo que compete a las liasas rompen enlaces carbono-carbono, carbono-oxigeno, carbononitrógeno y carbono-azufre. En la parte de enzimas sustrato es una molécula que sobre actúa en una enzima, el sustrato se une al sitio activo de la enzima, y se forma un complejo enzima-sustrato. El sustrato por acción de la enzima es transformado en producto y es liberado del sitio activo, quedando libre para recibir otro sustrato, a comparación de las ligasas que permiten la unión de dos moléculas, valiéndose de la energía obtenida por la degradación del ATP. Por lo tanto, general crean enlaces carbono-carbono, carbono-nitrógeno, carbono-oxigeno, carbono-azufre (Bermeo, 2019, citado en Alba et al., 2008).

Las enzimas proteolíticas conocidas también como peptidasas o proteasas, pueden utilizarse en la elaboración de variedad de productos y en diversas industrias, como los procesos catalizados por las enzimas siendo cada vez más numerosos, presentando una serie de ventajas frente a los catalizadores convencionales. ya que son proteínas formadas por uno o más cadenas de polipéptidos, trabajan en secuencia y catalizan ciento de procesos químicos, desnaturalizan proteínas y la convierten en energía según (Bermeo, 2019, citado en Mauricio et al., 2011).

Las frutas como la papaya, piña e higo presentan enzimas con actividad proteolítica. (Bermeo, 2019, citado en Yanza Guananga, 2010), comenta que debido a problemas de suministro de cuajo animal y la expansión de la industria de quesos forzaron la búsqueda de enzimas alternativas, por las amplias aplicaciones que tiene en la industria ha generado gran interés en la extracción de las enzimas con la finalidad de evaluar la mejor actividad enzimática.

La papaína es aplicada en las preparaciones enzimáticas crudas obtenidas del látex (Carica 
papaya) como distintas fracciones proteicas del mismo. La papaína y quimopaina son proteínas presentes en el látex (10 y $45 \%$ de la proteína soluble), el cual contiene también lisozima (20\%). La cisteína número veinticinco es esencial para la actividad de la papaína, y su oxidación por agentes oxidantes o iones de metales pesados causa inactivación indica (Bermeo, 2019, citado en Rivera Guerra, 2012).

La enzima bromelina estipula (Bermeo, 2019, citado en Parra et al., 2012) se obtiene a partir del jugo de la fruta o de los tallos de la piña (Ananas comosus). Es una glicoproteína del grupo de la cisteína proteasas. Actúa preferencialmente sobre aminoácidos básicos y aromáticos de las proteínas, su pH óptimo se encuentra en el rango de 5 a 8 . Tiene baja tolerancia térmica.

(Bermeo, 2019, citado en Carrera, n.d.) Describe que la enzima proteolítica ficina proveniente del látex del fruto verde, su actividad proteolítica se manifiesta al desnaturalizar sus proteínas sustrato mediante la ruptura de los enlaces disulfuro generados por aminoácidos sulfurados, es similar a la papaína que se extrae del látex de la papaya.

En Ecuador actualmente no existen muchas investigaciones sobre las enzimas proteolíticas de origen vegetal ya que actúan como catalizadores de reacciones metabólicas muchas de estas reacciones llegan a tener interés industrial debido a que su uso se está haciendo cada vez más importante.

La industria alimentaria en especial la que trabaja con sustratos lácteos debe ampliar los horizontes en lo que compete en la utilización de catalizadores biológicos, teniendo en cuenta que existen muchos de origen vegetal que son fáciles de obtener y que todavía no han sido utilizados por la industria lechera para la obtención de productos derivados de la misma.

Los trabajos que puedan derivarse del uso de enzimas vegetales son muy amplios tanto en las propiedades físicas, químicas, sensoriales y de cinética del funcionamiento teniendo en cuenta que se deben hacer combinaciones entre estas para poder obtener el sinergismo deseado en el producto alimentario.

Por lo registrado se establecen los siguientes objetivos:

- Determinar el rendimiento de látex obtenidos a partir de la papaya (Carica papaya), higo (Ficus carica), y piña (Annanas cosmosus L).

- Evaluar el rendimiento de las enzimas (Papaína, Ficina y Bromelina).

- Comparar la actividad de coagulación de las tres enzimas proteolíticas (Papaína, Ficina y Bromelina), obtenidos de tres frutas aplicado a la industria quesera.

\section{Metodología}

El desarrollo de la siguiente investigación se realizó en la Facultad de Ciencias Pecuarias y en la Facultad de Ciencias de la Escuela Superior Politécnica de Chimborazo, ubicada en la Panamericana Sur km $1 \frac{1}{2}$ en la ciudad de Riobamba, provincia de Chimborazo-Ecuador, utilizando para la obtención de las enzimas proteolíticas $5 \mathrm{~kg}$ de cada tipo de fruta (papaya, piña e higo), y para la evaluación de la actividad enzimática se utilizó 0,0375 g de enzima (papaína, 
Vol. 3, $\mathrm{N}^{\circ} 2.1$, p. 162-176, mayo, 2020

bromelina y ficina), por tratamiento y repetición, las unidades experimentales serán modeladas bajo un Diseño Completamente al Azar (DCA), que se ajustarán al siguiente modelo lineal aditivo.

Dónde

Valor del parámetro en determinación.

Efecto de la media por observación.

Efecto de los tratamientos.

Efecto del error experimental.

\section{Procedimiento experimental}

Las mediciones experimentales que se consideraron en esta investigación fueron:

\section{Características físico-químicas de los tres tipos de frutas}

Sólidos solubles (Método del Brixometro)

$\mathrm{pH}$ (Método potenciométrico)

\section{Métodos de obtención y preservación para las tres enzimas proteolíticas Método de ruptura mecánica (Enzima bromelina)}

Para la extracción y preservación de esta enzima se aplicó el método propuesto por (Bermeo, 2019, citado en Gallardo et al., 2008) donde la mejor condición para extraer la bromelina fue con el solvente etanol a una temperatura de $-10{ }^{\circ} \mathrm{C}$ y durante un tiempo de siete días, constando del siguiente procedimiento.

- Limpiar y desinfectar el área de trabajo y utensilios a utilizar para dicho proceso.

- Colocarse guates desechables.

- Lavar la fruta y pelar.

- Cortar en fragmentos pequeños

- Triturar con la ayuda de una licuadora.

- Tamizar

- Agregamos $1 \mathrm{ml}$ de etanol por cada $5 \mathrm{ml}$ de bromelina

- Congelar a $-10^{\circ} \mathrm{C}$ por 7 días.

- Descongelar.

- Centrifugar a 3500 rpm durante 10 minutos

Procesamiento del látex (Preservación de la actividad proteolítica)

- Colocar el látex extraído en bandejas de aluminio y congelar.

- Realizar el secado del látex utilizando un liofilizador a $60^{\circ} \mathrm{C}$ por 12 horas dependiendo de la cantidad del látex. Chequear cada 30 minutos.

- Una vez que el látex este granulado, el proceso de secado a finalizado.

- Se Coloca en refrigeración a $3{ }^{\circ} \mathrm{C}$ para un mejor mantenimiento de la enzima 
Método de extracción y preservación del látex mediante cortes horizontales y luego por centrifugación (Enzima papaína y ficina) como lo expone (Bermeo, 2019, citado en Mauricio et al., 2011)

- Limpiar y desinfectar el área de trabajo y utensilios a utilizar para dicho proceso.

- Colocarse guates desechables.

- Para la extracción del látex de la papaya y el higo se selecciona los frutos verdes y completamente desarrollados.

- Lavar las frutas de papaya e higo que van a ser utilizadas para el proceso.

- Secar las frutas con papel toalla para evitar posibles contaminaciones.

- Pesar las frutas para determinar el rendimiento del látex extraído una vez procesado.

- Realizar incisiones verticales de 1 a $2 \mathrm{~mm}$ de espesor en cada incisión para la extracción del látex.

- Colocar recipientes bajo la fruta para la recepción del látex.

\section{Procesamiento del Látex (Preservación de la actividad proteolítica)}

- Colocar el látex extraído en bandejas de aluminio y congelar.

- Realizar el secado del látex utilizando un liofilizador a $60^{\circ} \mathrm{C}$ por 12 horas dependiendo de la cantidad del látex. Chequear cada 30 minutos.

- Una vez que el látex este granulado, el proceso de secado a finalizado.

- Colocar el polvo en un mortero y molerlo hasta que se pulvericen.

- Se Coloca en refrigeración a $3{ }^{\circ} \mathrm{C}$ para un mejor mantenimiento de la enzima.

\section{Determinación de actividad enzimática}

Método de coagulación de la leche (Balls and Hoover)

Para la determinación de la coagulación de leche por el método de Balls and Hoover se realizó el método aplicado por (Bermeo, 2019, citado en Edwin \& Pablo, 2013) con algunas modificaciones donde menciona la capacidad que tiene la enzima en coagular la leche.

Los análisis se realizaron en 10 mililitros de leche entera a diferentes concentraciones de cada enzima $(0,0025,0,0050,0,01,0,02)$ gramos por mililitro de ácido acético, una vez preparada la solución de cada enzima con ácido acético se agregaron en los 10 mililitros de leche.

Se homogeniza en contenido y se controla el tiempo que demora hasta detectar la coagulación de la leche o lo formación de coágulos y se registra para cada uno de las cantidades y repeticiones. Según este autor los tiempos de coagulación deben estar entre 20 y 150 segundos para los resultados óptimos sin depender de la cantidad de enzima.

Para lo cual se aplicará la siguiente ecuación.

Dónde:

$\mathrm{V}=$ Velocidad

V máx $=$ Velocidad máxima

$\mathrm{S}=$ Sustrato

$\mathrm{Km}=$ Constante de Michaelis-Menten 
Vol. 3, N².1, p. 162-176, mayo, 2020

\section{Cantidad de proteína formado por unidad de tiempo}

Tiempos de coagulación

En los tiempos de coagulación se toma el tiempo en que la enzima se demora en reaccionar con la proteína de la leche hasta formar coágulos.

\section{Cantidad de enzimas necesarias a utilizar por medio} Fuerza o Actividad de Coagulación

Para determinar la fuerza de coagulación se utilizó el método de SOXHLET que dice la fuerza representa el número de volúmenes de leche coagulados por un determinado número de volúmenes de cuajo en 40 minutos a $35^{\circ} \mathrm{C}$. Se aplica la siguiente ecuación.

Dónde:

$\mathrm{F}=$ Fuerza de coagulación

$2400=$ Tiempo en segundos que normalmente la leche coagula a una temperatura de $35^{\circ} \mathrm{C}$.

$\mathrm{K}=$ Cantidad de leche en $\mathrm{ml}$.

$\mathrm{C}=$ Cantidad de cuajo en gramos.

$\mathrm{D}=$ Tiempo de coagulación en segundos

\section{Procedimiento}

- Se Tomó $10 \mathrm{ml}$ de leche entera y se colocó en un tubo de ensayo.

- Calentó en baño maría hasta una temperatura de $35^{\circ} \mathrm{C}$.

- Adicionar la respectiva cantidad de cuajo vegetal de papaína, bromelina y ficina.

- Controlar el tiempo que tardo en coagular la leche con cada una de las enzimas y sus diferentes cantidades, para poder calcular la fuerza de coagulación.

Característica físico-químico del medio luego de aplicado la enzima Contenido de proteína (Método Kjeldahl)

\section{Esquema del ADEVA}

El esquema del ADEVA que se utilizó en el presente trabajo de investigación se describe a continuación

Tabla 1: Esquema del experimento para la fruta

\section{TOTAL}

TRATAMIENTOCÓDIGOREPETICIONEST.U.E(Kg) Kg/trat

\begin{tabular}{|c|c|c|c|c|}
\hline (BROMELINA) & T1 & 5 & 5 & 25 \\
\hline (PAPAÍNA) & $\mathrm{T} 2$ & 5 & 5 & 25 \\
\hline (FICINA) & $\mathrm{T} 3$ & 5 & 5 & 25 \\
\hline Total, $g$ de fruta pa & $\operatorname{ara} n$ & $\mathrm{ra} \mathrm{en}$ & & 75 \\
\hline
\end{tabular}

T.U.E: Tamaño de la Unidad Experimental.

Fuente: Elaboración propia 
Vol. 3, $\mathrm{N}^{\circ} 2.1$, p. 162-176, mayo, 2020

Tabla 2: Esquema del experimento para la evaluación enzimática

\begin{tabular}{lcccc}
\hline \multicolumn{6}{r}{} & & & TOTAL \\
TRATAMIENTOCÓDIGO REPETICIONES T.U.E(mg) & mg/trat \\
\hline (BROMELINA) & T1 & 5 & 0,0375 & 0,1875 \\
(PAPAÍNA) & T2 & 5 & 0,0375 & 0,1875 \\
(FICINA) & T3 & 5 & 0,0375 & 0,1875 \\
Total, mg de fruta para muestra enzimática & & 0,5625 \\
\hline
\end{tabular}

T.U.E: Tamaño de la Unidad Experimental.

Fuente: Elaboración propia

\section{Resultados}

Análisis físico químico de los tres tipos de frutas. Solidos solubles (SST)

Al analizar en la (Tabla 3) el contenido de sólidos solubles totales de las diferentes frutas utilizadas en esta investigación se pudo conocer que, la piña, la papaya y el higo poseen valores de $\left(12,12^{\circ}\right.$ Brix, 7,62 ${ }^{\circ}$ Brix y 7,24 ${ }^{\circ}$ Brix) respectivamente donde existe diferencias altamente significativas $(<0,001)$, comparándolos con la norma técnica ecuatoriana (NTE INEN 2337, 2008) donde nos indica que la papaya debe tener un mínimo de $8^{\circ}$ Brix, la piña un mínimo de $10^{\circ}$ Brix, encontrándose dentro de los rangos establecidos sin tener mayor afectación, mientras que según él (CODEX STAN, 2005) el higo debe tener un mínimo de $18^{\circ}$ Brix, siendo el valor que más difiere con la presente investigación, esto puede deberse a lo mencionado por (Bermeo, 2019, citado en Gómez et al., 2018) en donde indican que la madurez es uno de los aspectos que refleja el comportamiento de los sólidos solubles o ${ }^{\circ}$ Brix, mientras que la cantidad de azucares en los frutos depende de la variedad de los mismos.

Tabla 3: Análisis físico químicas de los tres tipos de frutas

\begin{tabular}{llllllll}
\hline Parámetros & \multicolumn{3}{l}{ Características Físico químicas } & & \multirow{2}{*}{ EE. } & Prob \\
\cline { 2 - 6 } Solidos solubles & Papaya & Piña & & Higo & & & \\
Ph & 5,48 & A 12,12 & B & 7,24 & a & 0,37 & 0,001 \\
\hline
\end{tabular}

Realiza por: Elaboración propia

Fuente: INFOSTAT, 2019

EE. error estándar

Prob $>0,05$ : no existe diferencias estadísticas

Prob $<0,05$ : existe diferencias significativas

Prob $<0,01$ : existen diferencias altamente significativas

Medias con letras iguales en la misma fila no difieren estadísticamente de acuerdo a la prueba de uckey. 
pH

Al realizar el análisis del $\mathrm{pH}$ en los tres tipos de frutas higo, papaya y piña, existen diferencias altamente significativas $(<0,001)$ mostrando valores $(6,48,5,48$ y 2,20$)$ respectivamente, donde según la investigación de (Bermeo, 2019, citado en Badui \& Badui, n.d.) menciona que, todas las enzimas tienen un rango de $\mathrm{pH}$ óptimo para su correcto funcionamiento y para ser aplicadas en alimentos deben tener un valor entre 3 y 7, esto puede deberse a lo mencionado por (Bermeo, 2019, citado en Guacho, y otros, 2017, p 25) por lo que se puede determinar que la papaya y el higo se encuentran dentro de los establecido, siendo la piña la que se encuentra fuera del rango, debido a lo mencionado por (Bermeo, 2019, citado en Pérez et al., n.d.) donde señala que un pH por debajo de 3 es el resultado de la degradación enzimática por factores de obtención y almacenamiento.

\section{Análisis del rendimiento del látex de la fruta y la enzima.}

\section{Rendimiento del látex de la fruta (\%)}

Al analizar el rendimiento del látex en los tres tipos de frutas, se puede apreciar en la (Tabla 4) que existen diferencias altamente significativas, obteniendo valores de rendimiento de $(14,69 \%$ en la papaya, $11,81 \%$ del higo y $3,89 \%$ en la piña) esto puede deberse a lo mencionado por (Bermeo, 2019, citado en Villavicencio Marcial, 2011) donde indica que el estado de maduración de la fruta influye en la presencia de látex, obteniendo los mejores resultados en frutas en estado de maduración verde donde se encuentra mayor cantidad, facilitando la extracción de la enzima.

Tabla 4:_Análisis de la obtención de las tres enzimas proteolíticas.

\begin{tabular}{|c|c|c|c|c|c|c|c|c|}
\hline Parámetros & & & Fruta & & & & EE. & Prob \\
\hline & $\overline{\text { Papaya }}$ & & Piña & & Higo & & & \\
\hline $\begin{array}{l}\text { Rendimiento del látex } \\
\text { fruta }\end{array}$ & la 14,69 & $\mathrm{C}$ & 3,89 & $\mathrm{a}$ & 11,81 & $\mathrm{~b}$ & 0,42 & 0,001 \\
\hline Rendimiento de la enzil & na 13,61 & $\mathrm{~B}$ & 0,75 & $\mathrm{a}$ & 18,39 & $\mathrm{c}$ & 0,39 & 0,001 \\
\hline
\end{tabular}

Realiza por: Elaboración propia

Fuente: INFOSTAT, 2019

EE. error estándar

Prob $>0,05$ : no existe diferencias estadísticas

Prob $<0,05$ : existe diferencias significativas

Prob $<0,01$ : existen diferencias altamente significativas

Medias con letras iguales en la misma fila no difieren estadísticamente de acuerdo a la prueba de Tuckey

\section{Rendimiento de la enzima}

Los valores de rendimiento enzimático se encuentran en $18,39 \mathrm{~g} / \mathrm{Kg}$ para la ficina, $13,61 \mathrm{~g} / \mathrm{Kg}$ para la papaína y $0,75 \mathrm{~g} / \mathrm{Kg}$ para la bromelina, mostrándose diferencias altamente significativas $(<0.001)$, según lo investigado por (Bermeo, 2019, citado en Alejandra et al., 2012) que menciona, que el rendimiento se debe a factores genéticos y de cultivo, como también al método de extracción para cada una de ellas. 


\section{Análisis de la actividad enzimática}

Al realizar el análisis de la actividad enzimática se encuentran diferencias altamente significativas $(<0,001)$ como se muestra en la (Tabla 5), dando valores de (máx. 15,87 U/mg proto utilizando $0,0025 \mathrm{mg}$, min. 15,09 U/mg prot utilizando 0,005mg) para la papaína, (máx. 17,66 U/mg prot utilizando $0,0025 \mathrm{mg}$, min. $15,74 \mathrm{U} / \mathrm{mg}$ prot utilizando $0,02 \mathrm{mg}$ ) para la bromelina y para la ficina (máx. 16,19 U/mg prot utilizando $0,0025 \mathrm{mg}$, min. 14,84 U/mg prot utilizando $0,02 \mathrm{mg}$ ).

En estudios realizados por (Bermeo, 2019, citado en Edwin \& Pablo, 2013) menciona que los análisis de la actividad enzimática con el método de coagulación de leche (Balls and Hoover), obtuvieron una gama de tiempo de coagulación con la utilización de varias concentraciones de enzima, observando que a medida que aumenta la concentración de la enzima diluida en acido el tiempo de coagulación de la leche disminuye en los análisis realizados. Resultado que coincide con la presente investigación en lo que corresponde a la bromelina y ficina ya que a medida que va aumentando la concentración de la enzima el tiempo de coagulación va disminuyendo.

Al analizar la determinación de proteínas en la leche con el método de Kjeldahl, se obtuvo un valor de 2,92\% cumpliendo con lo establecido en la Norma Técnica Ecuatoriana (Bermeo, 2019, citado en NTE INEN, 10 n.d.) donde el contenido de proteína en la leche debe ser mínimo de 2,9 \%, encontrándose dentro de la normativa para ser utilizada en elaboración de productos lácteos.

Tabla 5: Análisis de la actividad enzimática de las tres enzimas proteolíticas.

\begin{tabular}{lllllll}
\hline DOSIS & & \multicolumn{3}{l}{ Cantidad } & & \\
& PAPAINA & & BROMELINA & \multicolumn{2}{c}{ FICINA } & \\
\cline { 1 - 5 } 0,0025 & 15,87 & C & 17,66 & d & 16,19 & d \\
0,005 & 15,09 & A & 17,26 & c & 16,01 & c \\
0,01 & 15,42 & b & 16,39 & b & 15,42 & b \\
0,02 & 15,79 & C & 15,74 & a & 14,87 & a \\
EE. & 0,03 & & 0,02 & & 0,04 & \\
Prob. & 0,001 & & 0,001 & & 0,001 & \\
\hline
\end{tabular}

Realiza por: Elaboración propia

Fuente: INFOSTAT, 2019

EE. error estándar

Prob $>0,05$ : no existe diferencias estadísticas

Prob $<0,05$ : existe diferencias significativas

Prob $<0,01$ : existen diferencias altamente significativas

Medias con letras iguales en la misma fila no difieren estadísticamente de acuerdo a la prueba de Tuckey.

\section{Análisis de los tiempos de coagulación}

Al analizar los tiempos de coagulación en las diferentes enzimas utilizadas en esta investigación se pudo conocer que los mejores resultad se obtuvieron con $0,005 \mathrm{mg}$ de papaína en un tipo de 61,44 seg, 0,02 mg de bromelina en un tiempo de 73,92 seg y con 0,02 $\mathrm{mg}$ de ficina un tiempo de $58 \mathrm{seg}$, presentando diferencias altamente significativas como se muestra en la (Tabla 6) esta diferencia puede deberse a lo mencionado por (Bermeo, 2019, citado en Alais, 2000) donde menciona que el tiempo de coagulación depende del volumen de cuajo como del volumen de leche 
a utilizar, este será medido en segundos y dependerá de la afinidad que tenga la enzima con el sustrato. Dando como mejor tiempo de coagulación a la ficina con una cantidad de 0,02 $\mathrm{mg}$ de enzima ya que coagula en menor tiempo de 58 segundos comparado con las otras enzimas y cantidades demostrando que es más proteolítica que la papaína y bromelina.

Tabla 6: Análisis de los tiempos de coagulación de los tres tipos de enzimas.

\begin{tabular}{|c|c|c|c|c|c|c|}
\hline \multirow{2}{*}{ DOSIS } & \multicolumn{6}{|c|}{ Cantidad } \\
\hline & \multicolumn{2}{|c|}{ PAPAINA } & \multicolumn{2}{|c|}{ BROMELINA } & \multicolumn{2}{|c|}{ FICINA } \\
\hline 0,0025 & 76,92 & $\mathrm{C}$ & 151,2 & $\mathrm{~d}$ & 84,84 & $\mathrm{bc}$ \\
\hline 0,005 & 61,44 & A & 126,12 & $\mathrm{c}$ & 67,32 & $a b$ \\
\hline 0,01 & 67,32 & $\mathrm{~b}$ & 90,84 & $\mathrm{~b}$ & 92,4 & $\mathrm{C}$ \\
\hline 0,02 & 75,12 & $\mathrm{C}$ & 73,92 & $\mathrm{a}$ & 58 & A \\
\hline EE. & 0,73 & & 0,67 & & 5,76 & \\
\hline Prob. & 0,001 & & 0,001 & & 0,001 & \\
\hline
\end{tabular}

Realiza por: Elaboración propia

Fuente: INFOSTAT, 2019

EE. error estándar

Prob $>0,05$ : no existe diferencias estadísticas

Prob $<0,05$ : existe diferencias significativas

Prob $<0,01$ : existen diferencias altamente significativas

Medias con letras iguales en la misma columna no difieren estadísticamente de acuerdo a la prueba de Tuckey

\section{Análisis de la fuerza de coagulación de la papaína, bromelina y ficina.}

En la investigación realizada por (Bermeo, 2019, citado en Juca Villalta, 2015) menciona que la fuerza de coagulación para las enzimas vegetales es menor que la fuerza de coagulación de la quimosina (cuajo comercial), debido a que tiene mayor especificidad para la caseína presentando un menor tiempo de coagulación, dando concordancia a los valores obtenidos en la presente investigación, siendo los mejores resultados $0,0025 \mathrm{mg}$ de papaína con $738432000 \mathrm{US}, 0,0025 \mathrm{mg}$ de bromelina con 1451520000 US, y $0,0025 \mathrm{mg}$ de ficina con 814464000 US. Como se muestra en la (Tabla 7) presentando diferencias altamente significativas.

Tabla 7: Análisis de la fuerza de coagulación de los tres tipos de enzimas.

\begin{tabular}{lllll}
\hline DOSIS & & $\begin{array}{l}\text { Cantidad } \\
\text { BROMELINA }\end{array}$ & & FICINA \\
\hline 0,0025 & PAPAINA & 1451520000,00 & D & $814464000,00 \mathrm{D}$ \\
0,005 & $294912000,00 \mathrm{C}$ & 605376000,00 & $\mathrm{C}$ & $323136000,00 \mathrm{c}$ \\
0,01 & $161568000,00 \mathrm{~B}$ & 218016000,00 & $\mathrm{~B}$ & $221760000,00 \mathrm{~b}$ \\
0,02 & $90144000,00 \mathrm{~A}$ & 88704000,00 & $\mathrm{~A}$ & $69600000,00 \mathrm{a}$ \\
EE. & 6531991,43 & 2920662,94 & & 14404109,4 \\
Prob. & 0,001 & 0,001 & & 0,001 \\
\hline
\end{tabular}

Realiza por: Elaboración propia

Fuente: INFOSTAT, 2019

EE. error estándar 
Prob $>0,05$ : no existe diferencias estadísticas

Prob $<0,05$ : existe diferencias significativas

Prob $<0,01$ : existen diferencias altamente significativas

Medias con letras iguales en la misma fila no difieren estadísticamente de acuerdo a la prueba de Tuckey

\section{Conclusiones.}

- Se determinó que el rendimiento de látex procedentes de los tres tipos de frutas para el higo es de $11,81 \%, 14,69 \%$ para la papaya y $3,89 \%$ para la piña.

- Se evaluó el rendimiento en las tres enzimas donde el mejor rendimiento es de $18,39 \mathrm{~g} / \mathrm{kg}$ para la ficina, $13,61 \mathrm{~g} / \mathrm{kg}$ para la papaína y $0,75 \mathrm{~g} / \mathrm{kg}$ para la bromelina.

- El mejor comportamiento enzimático y la velocidad de reacción es de 15, $09 \mathrm{U} / \mathrm{mg}$ prot para la papaína con un tiempo de coagulación de $61,44 \mathrm{~min} ; 15,74 \mathrm{U} / \mathrm{mg}$ prot para la bromelina con un tiempo de coagulación de 73,92 min y 14,87 U/mg prot para la fícina con un tiempo de coagulación $58 \mathrm{~min}$, siendo esta ultima la que presento un mejor comportamiento y velocidad enzimática.

- La necesidad de realizar combinaciones enzimáticas es imperiosa ya que queda demostrado que las velocidades de reacción entre las enzimas son diferentes por lo tanto la cantidad de las mismas varía.

- La calidad del sustrato siempre va a ser uno de los parámetros por los cuales la efectividad de la enzima tendrá una eficiente y eficaz actuación catalítica por lo que los análisis físicos y químicos son necesarios antes de realizar la catálisis enzimática

\section{Recomendaciones.}

- Realizar la limpieza y desinfección de la fruta para evitar contaminación en el látex y pérdidas en su actividad enzimática.

- Se recomienda utilizar temperaturas de 1 a $4{ }^{\circ} \mathrm{C}$ para la preservación del látex extraído de las frutas, evitando la desnaturalización enzimática.

\section{Referencias bibliográficas.}

Alais, C. (2000). Ciencia de la leche: principios de técnica lechera. https://books.google.es/books?hl=es\&lr=\&id=bW_ULacGBZMC\&oi=fnd\&pg=PR5\&dq $=$ Alais + principio + de + técnicas + lecheras\&ots $=Q$ N_s8_40dy\&sig $=4 \mathrm{sDZdQPGC8kdjWr4u}$ $\mathrm{yB} 3 \mathrm{tdprxe} 0 \# \mathrm{v}=$ onepage $\& \mathrm{q}=$ Alais principio de técnicas lecheras $\& \mathrm{f}=$ false

Alba, C., Díaz, M. F., Durán, E., Guerrero, K. L., \& Durán, J. (2008). Ciencia, tecnología e industria de alimentos (C. Alba, M. F. Díaz, E. Durán, K. L. Guerrero, \& J. Durán (eds.)). Grupo Latino Editores. http://biblioteca.unach.edu.ec/opac_css/index.php?lvl=notice_display\&id=2670

lejandra, P., Guerra, A., Fernanda, M., \& Avilés, Q. (2012). ESCUELA SUPERIOR POLITÉCNICA DEL LITORAL. 10. 
Badui, S., \& Badui, D. (n.d.). Química de los alimentos (P. EDUCACION (ed.); 4th ed.). Retrieved March 29, 2020, from www.pearsoneducacion.net

Carrera, J. E. (n.d.). PRODUCCIÓN Y APLICACIÓN DE ENZIMAS INDUSTRIALES PRODUCTION AND APPLICATION OF INDUSTRIAL ENZYMES.

CODEX STAN, 247. (2005). Norma General Del Codex Para Zumos (Jugos) Y Néctares De Frutas. http:/www.fao.org/fao-who-codexalimentarius/codex-texts/list-standards/es/

Dalgo, V. (2012). Obtención de un concentrado con bromelina a partir de la piña (Anananas comosus) y determinación de su actividad enzimática en sustratos proteínicos. 27. http://repo.uta.edu.ec/bitstream/123456789/3061/1/SBQ.27.pdf

Del Moral, S., Ramírez-Coutiño, L. P., De, M., \& García-Gómez, J. (n.d.). Aspectos relevantes del uso de enzimas en la industria de los alimentos. Retrieved May 11, 2020, from www.reibci.org

Edwin, A., \& Pablo, C. (2013). Extracción y estudio comparativo de las enzimas proteolíticas del fruto toronche (Carica-Stipulata) y de la papaya (Carica-Papaya) y su aplicación en la industria alimentaria. $\quad$ FIMCP, $20-22$. https://www.dspace.espol.edu.ec/handle/123456789/7532

Gallardo, L., Sánchez, A., Montalvo, C., \& Alonso, A. (2008). EXTRACCIÓN DE BROMELINA A PARTIR DE RESIDUOS DE PIÑA. In Ciencia y Tecnología de Alimentos (Vol. 18).

Gómez, M. I., Magnitskiy, S., \& Rodríguez, L. E. (2018). Potential yield and efficiency of N and $\mathrm{K}$ uptake in tubers of cvs. Capiro and Suprema (Solanum tuberosum Group Andigenum). Agronomía Colombiana, 36(2), 126-134. https://doi.org/10.15446/agron.colomb.v36n2.72766

Juca Villalta, D. N. (2015). UNIVERSIDAD DEL AZUAY FACULTAD DE CIENCIA Y TECNOLOGÍA ESCUELA DE INGENIERÍA EN ALIMENTOS. Universidad del Azuay.

Mauricio, R., Jaya, A., \& Ruales, J. (2011). Estudio de la variación de la actividad enzimática proteolítica del látex del babaco (Vasconcellea heilbornii cv babaco) en función de la edad del fruto. 35. https://bibdigital.epn.edu.ec/bitstream/15000/3924/1/CD-3656.pdf.

NTE INEN, 10. (n.d.). Leche pasteurizada, requisitos. Retrieved March 29, 2020, from https://181.112.149.204/buzon/normas/10-5.pdf

NTE INEN, 2337. (2008). NTE 2337 Jugos, pulpas, concentrados, néctares, bebidas de frutas y vegetales. Requisitos. https://archive.org/stream/ec.nte.2337.2008\#mode/2up Parra, A. Q., Clavijo, D., \& Martínez, 
Vol. 3, $\mathrm{N}^{\circ} 2.1$, p. 162-176, mayo, 2020

M. C. P. (2012). Cinética de la bromelina obtenida a partir de la piña perolera (Ananas Comosus) de Lebrija-Santander. Bistua: Revista de La Facultad de Ciencias Básicas, 10(2), 41-49. https://doi.org/10.24054/01204211.v2.n2.2012.84 Pérez, A., Carvajal, C., Torres, M., Medicinales,

M. M.-... P., \& 2006, undefined. (n.d.). Actividad proteolítica de extractos enzimáticos obtenidos de plantas de la familia Bromeliaceae. Scielo.Sld.Cu.

Retrieved March 29, 2020, from http://scielo.sld.cu/scielo.php?pid=S1028$47962006000200003 \&$ script $=$ sci_arttext\&tlng=en

Rivera Guerra, V. E. (2012). Evaluación de distintos cuajos naturales y procesados (Bovinos, Ovinos y Cuy) para la realización de queso fresco [Escuela Superior Politécnica de Chimborazo]. http://dspace.espoch.edu.ec/bitstream/123456789/1855/1/17T01083.pdf

Villavicencio Marcial, M. C. (2011). Extracción, concentración y cuantificación de la actividad enzimática de la papaína a partir de la papaya (carica papaya). 28-30.

Yanza Guananga, E. P. (2010). Utilización del látex de las hojas, tallo y frutos de la papaya (tipo Hawaiana) como coagulante Natural en la elaboración de queso fresco. ESPOCH.

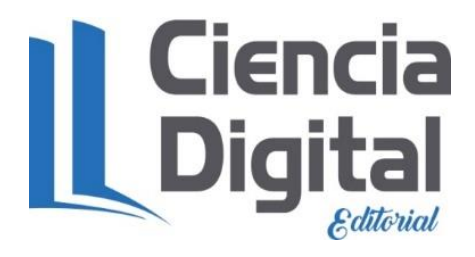


PARA CITAR EL ARTÍCULO INDEXADO.

Bermeo Berrones, J. G., Salgado Tello, I. P., Flores Mancheno, C. I., \& Sánchez Herrera, T. E. (2020). Evaluación da la actividad de tres enzimas proteolíticas como biocatalizadores lácteos. ConcienciaDigital, 3(2.1), 162-176. https://doi.org/10.33262/concienciadigital.v3i2.1.1231

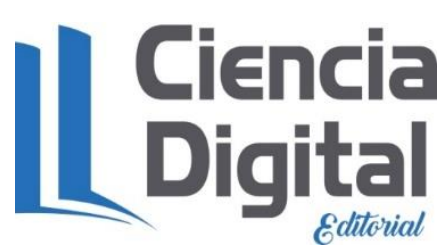

El artículo que se publica es de exclusiva responsabilidad de los autores y no necesariamente reflejan el pensamiento de la Revista Conciencia Digital.

El artículo queda en propiedad de la revista y, por tanto, su publicación parcial y/o total en otro medio tiene que ser autorizado por el director de la Revista Conciencia Digital.

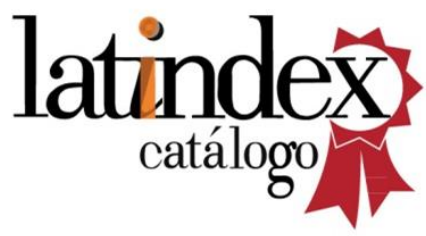

Conciencia

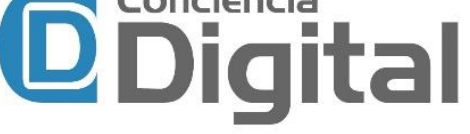

